\title{
PSYCHOLOGICAL MONITORING OF OVERTRAINING AND STALENESS
}

\author{
W. P. MORGAN, EdD, FACSM, D. R. BROWN, PhD* , J. S. RAGLIN, MS, P. J. O’CONNOR, MS and \\ K. A. ELLICKSON, PhD \\ Sport Psychology Laboratory, University of Wisconsin-Madison
}

\begin{abstract}
It is widely agreed that overtraining should be employed in order to achieve peak performance but it is also recognised that overtraining can actually produce decrements in performance. The challenge appears to be one of monitoring stress indicators in the athlete in order to titrate the training stimulus and prevent the onset of staleness. The present paper summarises a ten-year research effort in which the mood states of competitive swimmers have been monitored at intervals ranging from 2-4 weeks during individual seasons for the period 1975-1986. The training cycle has always involved the indoor season which extends from September to March and the athletes who served as subjects were 200 female and 200 male competitive swimmers. The results indicate that mood state disturbances increased in a dose-response manner as the training stimulus increased and that these mood disturbances fell to baseline levels with reduction of the training load. Whilst these results have been obtained in a realistic setting devoid of experimental manipulation, it is apparent that monitoring of mood state provides a potential method of preventing staleness.
\end{abstract}

Key Words: Overtraining, Staleness, Mood states, monitoring.

\section{INTRODUCTION}

It is widely accepted by exercise scientists, coaches and athletes alike, that success in endurance events requires progressive increases in the training stimulus and it appears that overtraining is a prerequisite for peak performance in the elite athlete. It is recognised, however, that overtraining can lead to staleness and recent research involving elite distance runners has revealed that $64 \%$ of the female (Morgan et al, in press) and $66 \%$ of the male (unpublished data) athletes who were studied had experienced staleness at some point during their competitive careers. Whilst an intense training session will produce acute fatigue, the athlete normally recuperates within 12-24 hours. There are times, however, when the athlete does not recover following a night's sleep and a day of rest. The athlete may reach a point where he or she is unable to initiate or complete one or more scheduled microcycles $^{2}$ because of chronic fatigue and at this point performance in competition usually falls below expected or customary levels.

Coaches and sport scientists in the United States have historically labelled this state as staleness (Griffith, 1926; Karpovich, 1959; Morehouse and Rasch, 1958; Morgan, 1985b, Wolf, 1971), whereas European workers have tended to use the term overtraining to describe this problem (Bompa, 1983; Harre, 1982; Kereszty, 1971; Mellerowicz \& Barron, 1971). Also, this state is sometimes referred to as overfatigue or overstrain (Czajkowski, 1982) and some authors have used the terms overtraining and staleness synonymously (Parmenter, 1923). These terms should not be so employed, however, since staleness represents an

Address for correspondence:

William P. Morgan, EdD

Professor and Director

Sport Psychology Laboratory

2000 Observatory Drive

University of Wisconsin-Madison

Madison, Wisconsin 53706

USA

*Present address:

Department of Health, Physical Education and Recreation

Miami University

Oxford, Ohio

USA outcome or product, whereas overtraining reflects a process. Furthermore, the term overtraining will be employed in this article as a stimulus or antecedent variable and staleness will be used as a response or consequent variable. A schematic depicting this relationship appears in Fig. 1 and it has proven heuristic to conceptualise the problem in this manner for several reasons. First, the idea of viewing overtraining as a process and staleness as the state of being stale, has intuitive appeal. Second, in endurance sports such as distance running, swimming, cycling, and cross-country skiing, overtraining is approached as a deliberate, planned, and appropriate feature of the monocycle. ${ }^{2}$ Also, the training stimulus (e.g. distance per day) can be quantified and this permits "dose-response" evaluations and modelling efforts.

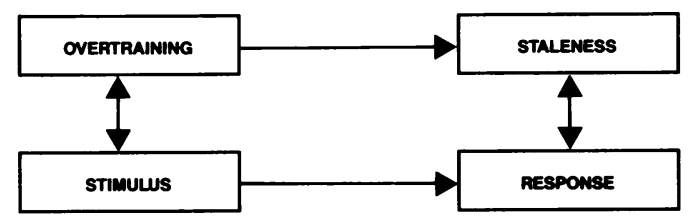

Fig. 1: Schematic depicting the distinction and inter-relationship between overtraining and staleness.

By comparison, staleness is viewed as undesired and pathological. Once the state or syndrome ${ }^{3}$ known as staleness is diagnosed, it must be dealt with by the sports medicine team but prevention of this state is far more desirable than treatment. Indeed, with the exception of reduced training or complete rest, there is no accepted treatment for this problem at the present time. It is also noteworthy that physicians, physiologists and psychologists alike have all recommended rest as the

'This paper was presented by the first author as the Inaugural Lecture at the opening of the London Sports Medicine Institute, Medical College of St. Bartholomew's Hospital, 11 December, 1986.

2Bompa (1983), Harre (1982), and Matveyev (1981) refer to an entire year's training programme as the mono-cycle, the indoor or outdoor season as the macro-cycle (e.g., September-March), and sub-units of the macro-cycle as the micro-cycle, and there could be 10-30 micro-cycles within a given indoor season. These terms and definitions have been employed in this report.

3/t may not seem appropriate to accord syndromic status to the state known as staleness, but the authors believe that this particular problem resembles recognised syndromes such as endogenous or primary depression (Akiskal and McKinney, 1975). 
treatment for staleness since the early 1920s (Griffith, 1926; Parmenter, 1923).

\section{SUMMARY}

It is widely agreed that overtraining should be employed in order to achieve maximal performance levels in many sports but it is also recognised that overtraining can actually produce decrements in performance. The challenge appears to be one of monitoring stress indicators in the athlete in order to titrate the training stimulus and prevent the onset of staleness. The primary purpose of the present paper will be to summarise a series of investigations carried out in the Sport Psychology Laboratory at the University of Wisconsin-Madison over the past ten years. While physiological and psychological data have both been collected in this research, the present paper will focus on the psychological data.

This systematic research effort has shown that it is relatively simple to employ training loads that overload most of the athletes on a given team. While this approach consistently improves performance in many athletes, it is also recognised that an overtraining stimulus will produce staleness in some individuals. It is very easy, of course, to prevent staleness by simply not training hard but this is not a realistic approach given the emphasis placed on overtraining in most programmes. The data collected in the present investigation demonstrate that monitoring of mood states during training offers considerable potential for prevention of staleness. Whilst our results have been obtained primarily from research involving swimmers involved in a competitive university programme, there is limited evidence that the same type of response occurs in other sports. The remainder of this paper deals with a summary of this research effort.

\section{GENERAL PROCEDURES}

\section{Psychometric Assessment}

The Profile of Mood States (POMS) (McNair et al, 1971) has been administered to approximately 200 men and 200 women who have been members of the University of Wisconsin-Madison swimming teams during the period 1975-1986. In some cases the POMS was administered at the outset of the season, following the peak micro-cycle in mid-season, and again at the end of the season. For other seasons the POMS was administered more frequently at periods ranging from every two to four weeks.

The POMS contains 65 items and it yields a global measure of mood, as well as measures of tension, depression, anger, vigour, fatigue and confusion. In most of the examples cited in this paper, the global measure of mood has been reported. A global score is computed by adding the five negative mood states (tension, depression, anger, fatigue, and confusion) and subtracting the one positive mood state (vigour). Since this computational procedure sometimes yields negative values, we have elected to add a constant of 100 in most of the examples reviewed in this paper. In other words, an individual with a global mood score of +25 on the POMS would be scored as 125 , whereas a person with a score of -25 would be scored as 75. While the POMS has four possible response sets, we have employed the standard instructional set in this research. That is, the subject has been asked to respond in terms of "how you have been feeling during the past week including today" rather than "generally", "today", or "right now" (McNair et al, 1971).

\section{Training Loads}

The swimmers who have served as subjects in the analyses reported in this paper have been exposed to differential levels of training stress during the mono-cycle (1 Sep- $D$ 31 Aug), as well as the macro-cycle (1 Sep-1 Mar). Furthermore, the training load during any given micro-cycle (e.g. 1 Dec-1 Jan) has varied considerably. In other words, these swimmers have been studied within a realistic setting as opposed to one in which the training load has been manipulated experimentally.

The average training load, measured in yards per day, is summarised in Fig. 2 for a recent season. Whilst the specific peak for any given season, as well as the taper (i.e. reduced training) has varied somewhat, this sample training log can be viewed as representative of the approach taken in this programme.

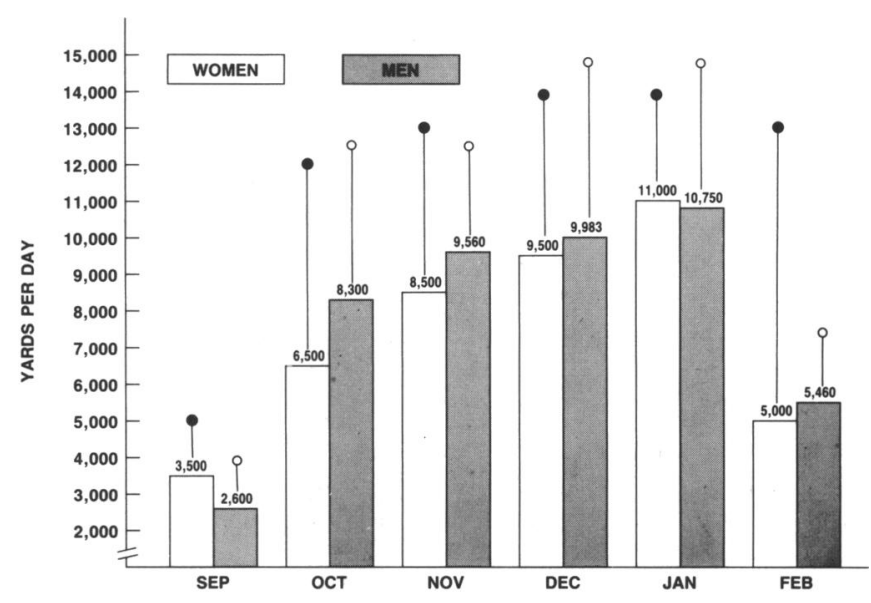

Fig. 2: Training Load in yards per day for a representative season.

The women $(N=22)$ and men $(N=31)$ included in this analysis did not differ significantly in terms of average yards per day during the macro-cycle. The average training load was approximately 3,000 yards per day at the outset and increased to a peak load of about 11,000 yards per day. This peak load was followed by a taper to 5,000 yards per day just prior to the conference championship. The bands extending above each bar represent the highest training load achieved for a given swimmer and it is emphasised that major individual differences existed throughout all phases of the macro and micro-cycles. Over a period of ten years it has been observed that the greatest amount of mood disturbance occurs in late January following the most intense micro-cycle which has averaged 10,000 to 15,000 yards per day. This peak training load is accomplished with twice daily training and it is not uncommon for $5-10 \%$ of the swimmers to experience what we regard as staleness at this time. Again, we defined staleness as a state characterised by reduced performance in concert with an inability to train at customary levels. During this research we have routinely referred athletes with staleness to our counselling psychology and out-patient psychiatry services and approximately $80 \%$ of these individuals have been judged to possess depression of clinical significance.

There is an absence of epidemiological research dealing with the incidence of staleness in swimmers but it is unlikely that the focus of this paper can be generalised to swimmers participating in programmes characterised by lower peak loads (e.g. 3,000 to 5,000 yards per day). In other words, it is our belief that staleness represents a potential problem in programmes which incorporate overtraining micro-cycles in the range of 10,000 to 15,000 yards per day. 


\section{Perception of Training Load}

In an effort to quantify the perception of training intensity, we have administered a 7-point psychophysical category scale to our swimmers throughout the season. This scale is illustrated in Fig. 3, and the swimmers were asked prior to a given training session to rate the intensity of the previous day's work-out.

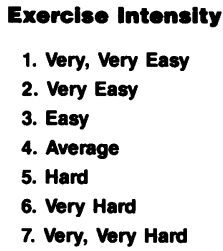

Fig. 3: Psychophysical category scale used in rating the intensity of training sessions.

Results for 10 female and 18 male swimmers are summarised in Fig. 4, and the findings for this particular macro-cycle ${ }^{2}$ are representative for those observed in other years. It will be noted that perception of exercise intensity increased significantly in both groups during mid-season and this particular macro-cycle included the peak training load. It will also be noted in Fig. 4 that tapering resulted in a mean rating somewhat below that observed in early season. Thus, perception of effort was found to covary with titration of the training load.

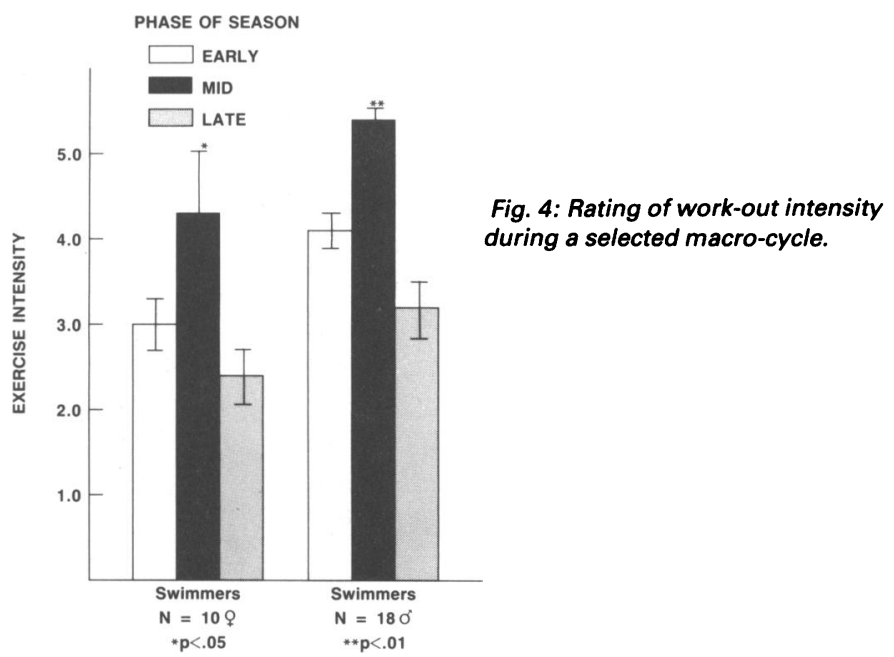

Many coaches believe that swimmers "feel faster" if they are first overtrained and then tapered. It is very important to recognise, however, that tapering will not necessarily return a swimmer to his or her baseline levels in terms of mood state or performance. Furthermore, the results depicted in Fig. 4 represent the collective response patterns for two groups of swimmers and there were individual swimmers who did not respond in this manner.

\section{MONITORING OF MOOD STATE}

\section{Study 1}

In the first investigation the POMS was administered to a sample of 16 male swimmers at the beginning, middle and close of a given indoor season. The results are summarised in Fig. 5, and it was found that global mood disturbance increased significantly ( $p<0.01$ ) in mid-season during the micro-cycle which included peak training. Following a taper the global mood score returned to the baseline level recorded during the initial micro-cycle. Again, however, it is emphasised that these results hold for the group but there were individual swimmers $(N=2)$ who did not respond to the taper.

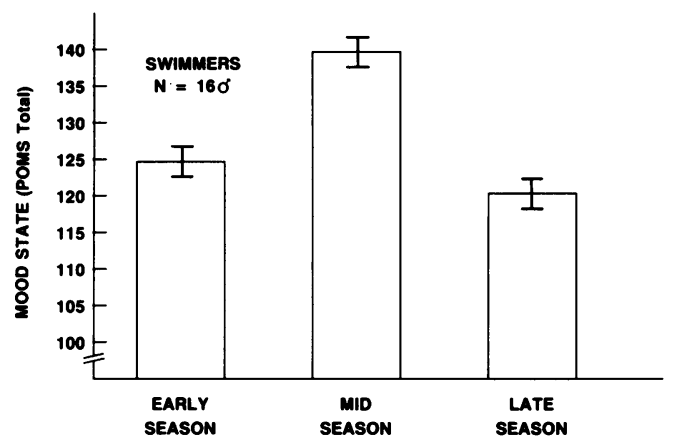

Fig. 5: Global mood state at the outset, middle, and close of a selected macrocycle.

Inspection of the individual POMS scales revealed that the significant changes in global mood were due to a significant increase in fatigue $(P<0.01)$ and a significant decrease in vigour $(P<0.01)$. These results are summarised in Fig. 6. In other words, the alterations in variables such as depression and tension were not significant for this group. Nevertheless, two of the swimmers experienced significant increases in depression and tension with peak training. It is probable that alterations in fatigue and vigour are precursors of more serious mood disturbances such as depression or chronic anxiety.

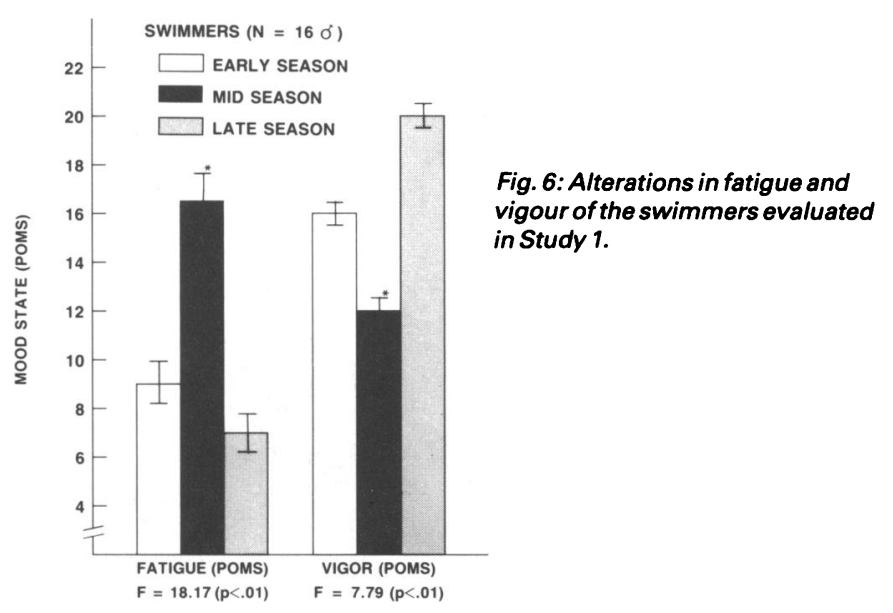

Study 2

In a separate analysis involving 15 female swimmers who were tested psychologically in September and again following an overtraining stimulus in January, it was observed that a significant disturbance in global mood took place. However, in this case evaluation of the individual POMS variables revealed a significant increase in depression $(p<0.01)$ and anger $(P<0.01)$. These results are summarised in Fig. 7. While vigour decreased as it had in the previous study involving male swimmers, this decrease was not significant $(P>0.05)$. This could reflect a gender difference but it is unlikely since this has never been observed in any of our subsequent comparisons of male and female swimmers.

Perhaps the most notable feature of this particular study is the apparent paradox observed in connection with the exercise-depression relationship. Whereas vigorous exercise is known to reduce depression in moderately depressed individuals (Morgan, 1985a; Morgan \& Goldston, 1987), depression also seems to be a product of 


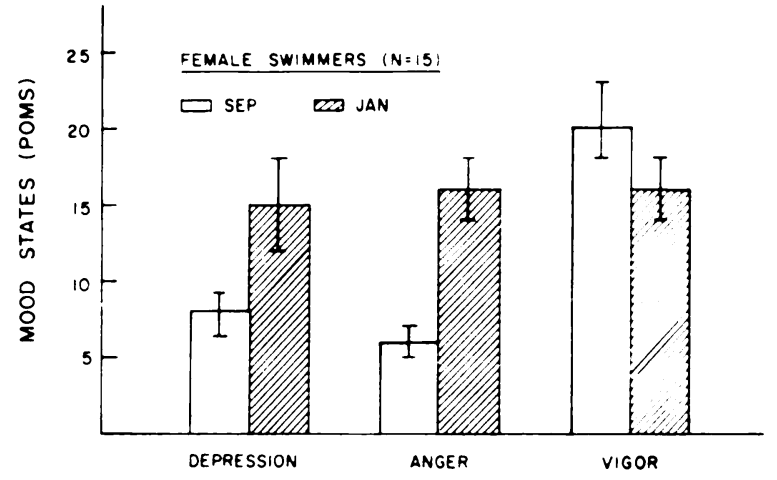

Fig. 7: Alterations in mood state for women swimmers following overtraining.

overtraining. Further, the symptoms presented by the stale athlete are remarkably similar to many of those seen in primary or endogenous depression (Akiskal and McKinney, 1975). Stale athletes, for example, are often characterised by: physiological and psychomotor retardation, chronic fatigue, depressed appetite, weight loss, insomnia, decreased libido, muscle soreness and elevated depression and tension. Many of these changes are understandable given the widespread reports of metabolic (Foster et al, 1982), hormonal (Hakkinen et al, 1985; Hickson et al, 1979; Stray-Gundersen, 1986), muscular (Dressendorfer and Wade, 1983), hypothalamic (Barron et al, 1985) and cardiovascular (Czajkowski, 1982; Dressendorfer et al, 1985) changes consequent to an overtraining stimulus. Indeed, one of our test subjects, a 20-year-old male swimmer, experienced a documented myocardial infarction during training (Hanson et al, 1982).

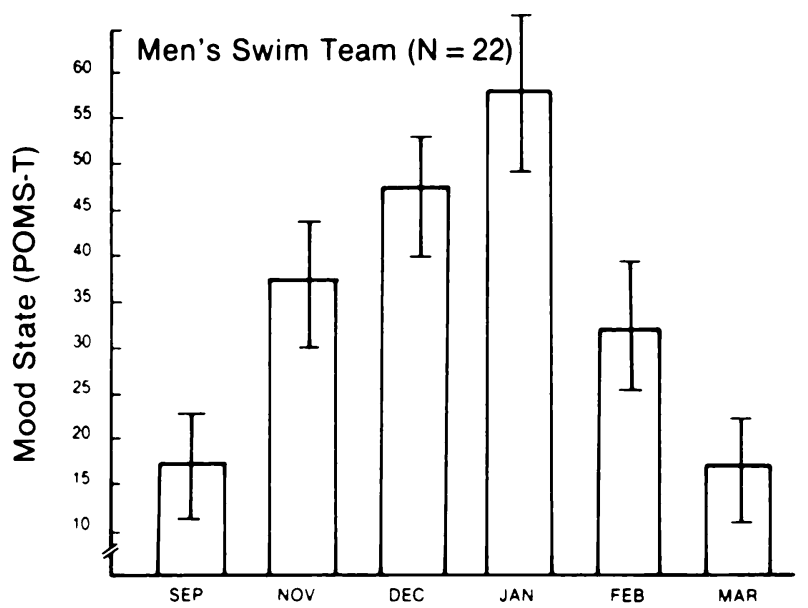

Fig. 8: Global mood state in male swimmers at various points during a selected macro-cycle.

\section{Study 3}

In a subsequent study, 22 male and 18 female swimmers were administered the POMS throughout a selected macrocycle. The results for the males are summarised in Fig. 8 . It will be noted that a stepwise increase in mood disturbance occurred with progressive increases in training load, and reduction of the training load from approximately 13,000 yards per day to 4,000 yards per day resulted in a return of global mood state to baseline levels. The data summarised in Fig. 8 suggests that a dose-response effect took place. That is, mood disturbance and improvement was directly related to titration of the training load. Again, however, it should be emphasised that major individual differences existed.

The data for the women's team are summarised in Fig. 9 and these results are identical with those reported for the

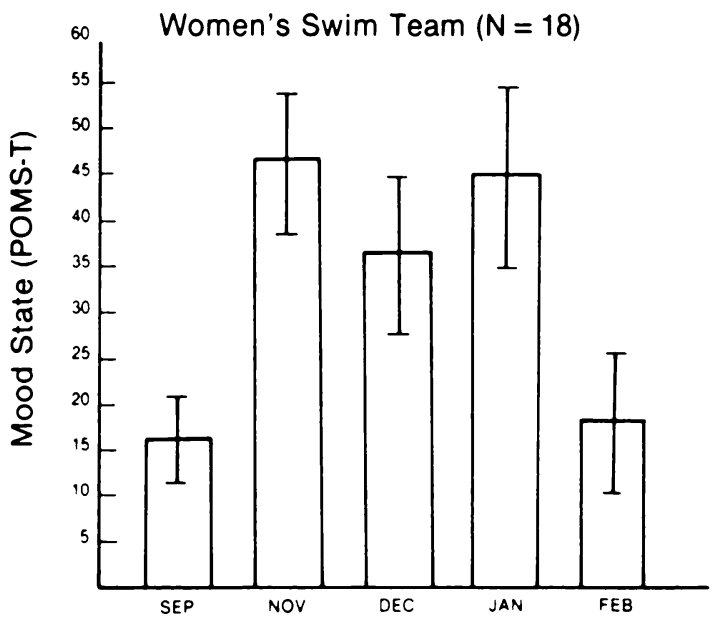

Fig. 9: Global mood state in female swimmers at various points during a selected macro-cycle.

men's team with one important exception. It was anticipated that mood disturbance would increase to a peak level in December but it will be noted that global mood improved slightly at this point. This apparent contradiction can be readily explained and the explanation serves to emphasise an important principle. In this particular case the coach elected to reduce the training load for a period of several days during the month of December in order to peak for an important dual meet. The resulting decrease in global mood was not significant $(P>0.05)$, but this reduction in training load resulted in enhanced performances. It has been reported that performance increases following a taper (Bompa, 1983; Harre, 1982; Matveyev, 1981), and it is noteworthy that mood state is responsive to brief manipulations of the training load.

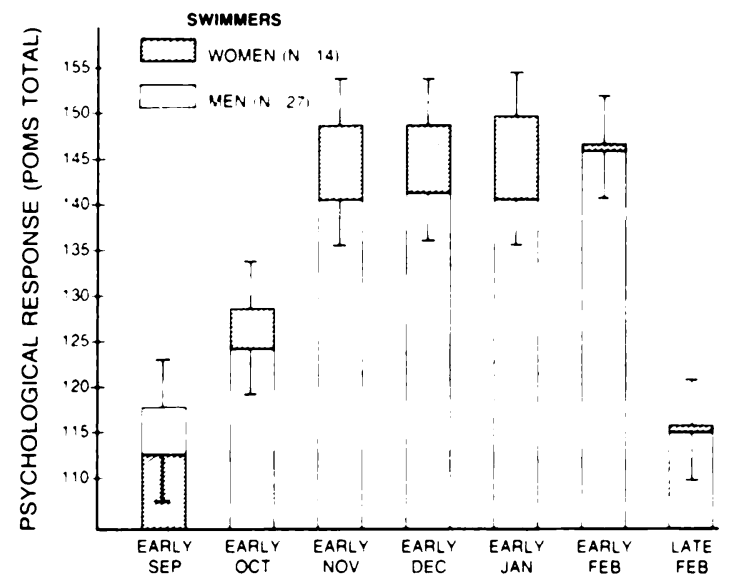

Fig. 10: Global mood states of female and male swimmers at various points during a selected macro-cycle.

\section{Study 4}

In a later investigation the POMS was administered to 14 female and 27 male swimmers across a macro-cycle consisting of 6 months. The results of this study are summarised in Fig. 10. These data were analysed by means of a repeated measures ANOVA for multifactor experiments. There was a significant $(P<0.01)$ mood disturbance which took place during the overload microcycles and the return to baseline levels following the taper was also significant. The $F$ ratio for gender was not significant and this finding indicates that overtraining and tapering had the same effect on the mood states of female and male swimmers.

During the ten year period that we have systematically monitored the swimmers in these programmes, the 
coaches have adopted different tapering strategies from time to time. In this case the coaches had elected to employ a rapid, rather than a gradual taper. The data in Fig. 10 seem to suggest that mood state returned to baseline with only several weeks of tapering.

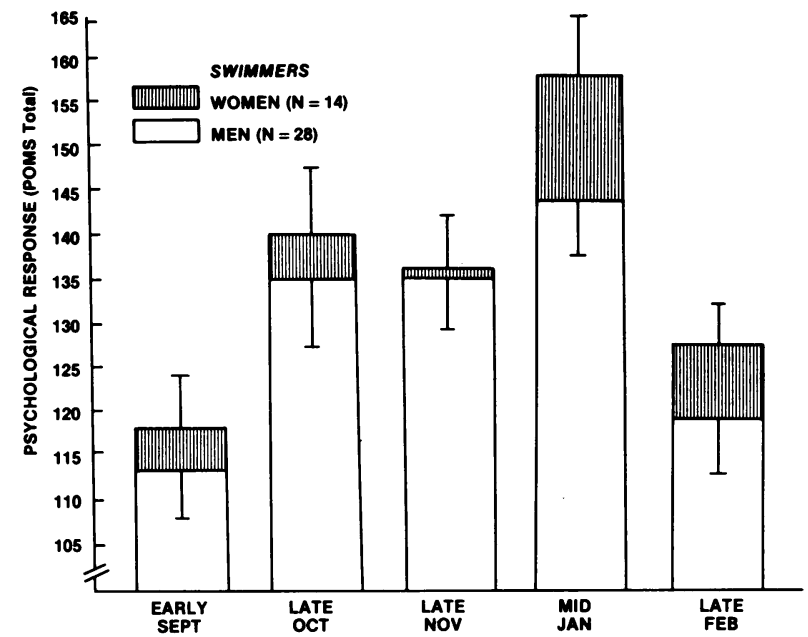

Fig. 11: Global mood states of female and male swimmers at various points during a selected macro-cycle.

\section{Study 5}

The same procedures and analyses were performed in a fifth investigation involving female $(N=14)$ and male $(N=$ 28) college swimmers and the results are summarised in Fig. 11. The overall findings are essentially the same as those noted in the previous study summarised in Fig. 10. Calculation of a total mood score on the basis of the POMS scores $(T+D+A+F+C+100-V)$ yielded a mean score of approximately 115 for the women's and men's teams at the beginning of training in early September. Increased training provoked a significant increase in mood disturbance to mean levels of 135 and 140 for the men and women respectively. These values were increased to mean levels of 144 and 156 for the men and women respectively in midJanuary following the most intense micro-cycle and both groups experienced significant decreases (119-126) following the taper period in late February. The global mood state values in late February prior to championship competition were essentially the same as those observed in early September at the outset of the macro-cycle. The differences in mood state scores for the men and women in this study were not statistically significant during any of the micro-cycles evaluated.

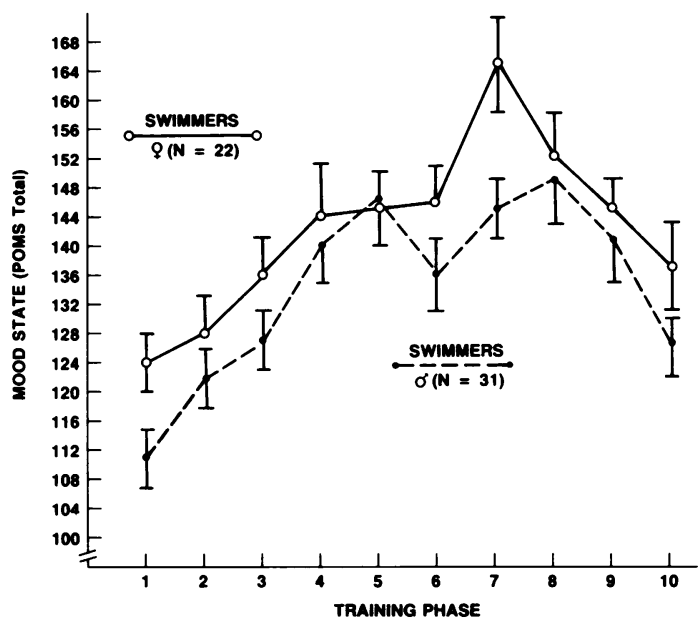

Fig. 12: Global mood states of female and male swimmers at various points during a selected macro-cycle.

\section{Study 6}

The results described in the above study were replicated in a subsequent investigation involving 22 female and 31 male swimmers. The swimmers completed the POMS during 10 micro-cycles within a macro-cycle that extended from early September to early March. The results are summarised in Fig. 12 and it was observed that global mood disturbance increased significantly $(P<0.01)$ during the first 5 microcycles in both groups. The male swimmers continued to increase through the seventh micro-cycle, whereas the female swimmers did not differ significantly between the fifth and eighth micro-cycles. Both groups experienced an improvement in mood state during the taper but neither group returned to their baseline levels at the time of championship competition. In this particular example it can be seen that recovery from overtraining was not optimal from a mood state perspective.

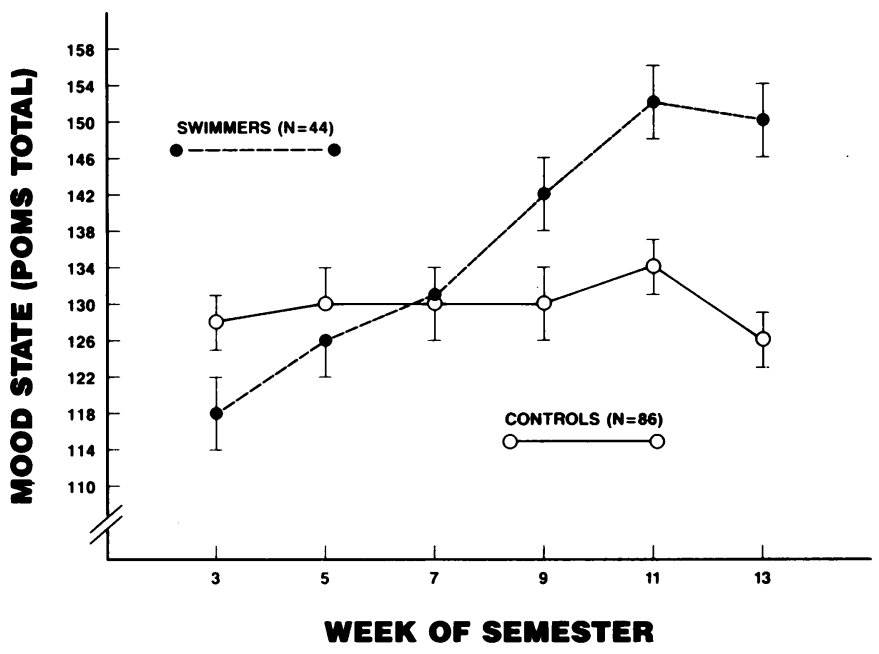

Fig. 13: Global mood state of competitive swimmers and non-athlete controls during a 13-week period.

\section{Study 7}

It is possible that many of the changes in mood state described in this paper might be independent of training for swimming competition. The observed psychological effects might occur in college students in general because of exposure to various academic, economic and social stressors. For this reason an investigation was carried out with 44 swimmers and 86 controls during a thirteen week period which extended from early September through early December. The swimming and control groups both consisted of male and female subjects, and since preliminary analyses indicated and absence of gender differences, the sub-groups have been combined for illustrative purposes. The results are summarised in Fig. 13. It will be noted that the swimmers experienced a significant mood disturbance from the fifth to the eleventh week of the semester, whereas the controls did not differ significantly at any point throughout the 13-week period. Furthermore, while the swimmers scored significantly lower $(P<0.05)$ than the controls on the POMS at the outset of the semester, this difference was erased during the fifth to seventh weeks, and the swimmers scored significantly higher $(P<0.01)$ than the controls during weeks 9 through 13. These findings offer additional evidence to support the view that increased mood disturbance with overtraining is associated with the training stimulus per se rather than other stressors common to college students in general. 


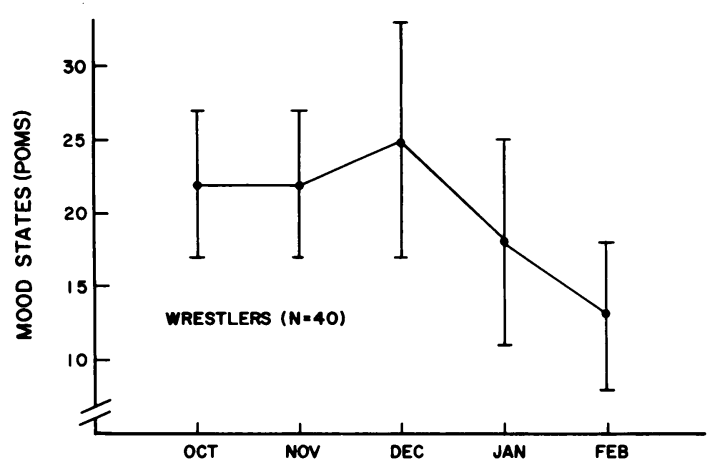

Fig. 14: Global mood state in 40 wrestlers during a selected macro-cycle.

\section{Study 8}

In an effort to evaluate whether or not the psychological effects observed with swimmers could be generalised to other sports, we elected to study a group of wrestlers involved in an intense training programme. The subjects consisted of $\mathbf{4 0}$ members of the University of WisconsinMadison Wrestling Team and they were evaluated throughout a selected macro-cycle. The results are summarised in Fig. 14. It was not possible to evaluate this group in early September because of logistical problems but, other than the apparent elevation at the time of the pretest, the results parallel those reported for the swimmers. A repeated measures ANOVA indicated that these mood state fluctuations were not significant $(P>0.05)$ and this is undoubtedly due to the variability in responses. Nevertheless, the results are quite similar to those observed in each of the swimming cohorts. That is, mood disturbance reached its highest level in December following the most intense micro-cycle and tapering of training load resulted in improved mood state in late February prior to championship competition.

Four of the wrestlers $(10 \%)$ developed staleness during this particular season. One of these individuals elected to retire from competitive wrestling and he never resumed training. The remaining three were offered therapy and rest. All three elected to rest for periods ranging from one to two weeks and this is noteworthy since absence from team training and competition in this setting requires that an individual regain (i.e. "wrestle-back") his former position when returning. One of the three individuals took part in psychotherapy and his therapy continued after he returned to the team. All three individuals experienced improved performances according to the coaching staff and two of these individuals achieved national rankings at the close of the season.

The trend reflecting improved mood which accompanied the reduction in training load, while not statistically significant, was certainly in the desired direction, and the effect of complete rest in the three cases described above also speaks in favour of rest as a recuperative strategy. Unfortunately, the athlete often does not have the luxury of time; that is, tapering and/or rest is not possible because the athlete is confronted with championship competition and insufficient time to taper.

The substantial variability in these data resulted from selected athletes scoring two and three standard deviations above and below the group means during various microcycles. It appears that some wrestlers, just like some swimmers, are particularly susceptible to overtraining, while others are seemingly immune.

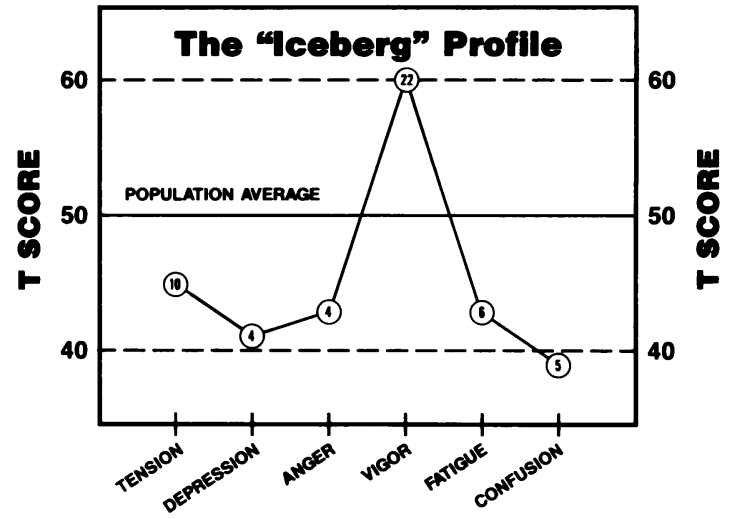

Fig. 15: The "iceberg profile" of a selected swimmer at the outset of a macrocycle.

\section{THE ICEBERG PROFILE}

It has previously been reported that elite athletes, as well as active individuals in general, tend to score below the population average on the tension, depression, anger, fatigue and confusion scales of the POMS and they usually score about one standard deviation above the population average on vigour (Morgan, 1985b). These findings have been consistently noted for swimmers, runners, rowers and wrestlers. This particular profile is illustrated in Fig. 15 for a selected male swimmer who was tested in early September at the outset of a macro-cycle. This profile has been called the "iceberg profile" because the resulting configuration resembles an iceberg; that is, the negative mood states all fall below the population average ( $T$ Score of 50 ), and the one positive mood state (vigour) falls one standard deviation above the population mean. This particular swimmer's total or global mood score was 107 at the outset of training (i.e. $T+D+A+F+C+100-V=10+4+4+6+5+$ $100-22=107$ ). This value can be employed as a baseline for this particular swimmer who experienced staleness during a later micro-cycle. He reached the point where he was unable to complete regularly scheduled work-outs. He

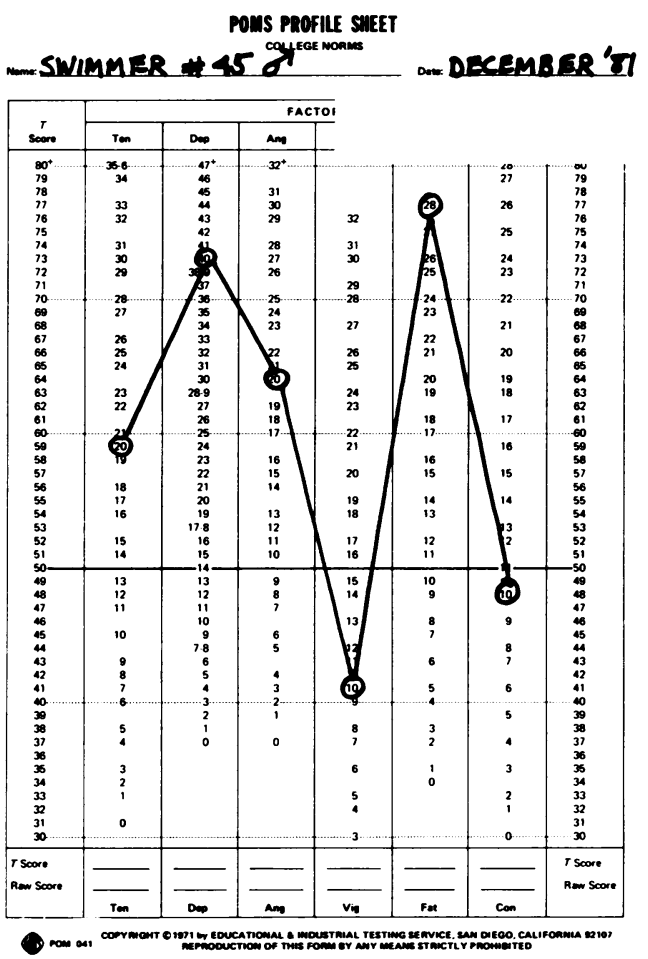

Fig. 16: Inverted "iceberg profile" of the swimmer depicted in Fig. 15 following overtraining which resulted in staleness. 
eventually reached the point where he was suffering from chronic fatigue to the extent that he was not able to begin, much less complete, a work-out session. His POMS profile at this point differed dramatically from his original iceberg profile and it is depicted in Fig. 16 on a standard POMS profile sheet. It is obvious that this particular swimmer changed significantly and computation of his total mood score yielded a value of $208(20+40+20+28+10+100-10)$ at this point. This swimmer was subsequently diagnosed as depressed by a clinical psychologist and it will be noted that his depression score on the POMS increased from a raw score of 4 to one of 40 . This represented a shift from a Tscore of 41 to one of 73 . This individual was rested for a period of one week and he resumed training at a greatly reduced level. His psychotherapy was of a short-term nature and he returned to excellent form, placing high in the conference standings at the conclusion of the season. Additional cases will not be presented but we wish to emphasise that the course of overtraining, staleness and recovery seen in this swimmer is representative of other cases we have been involved with during the past ten years.

\section{TAPERING}

Rather than using complete rest to treat the problem of staleness, it is possible to simply reduce the training load. This can be particularly effective if there is sufficient time available to develop a tapering plan or schedule. The process of overtraining, staleness, tapering and recovery can be illustrated with a case study. The athlete was a 22year-old competitive swimmer at the time of testing and he was exposed to a training load of 4,000 yards per day during the first micro-cycle. At this particular point in time his total POMS score was 106 . With an increase in the training load to 11,000 yards per day, the total POMS score increased to 165. These results are summarised in Fig. 17, and it will be noted that the POMS total score increased to 183 following

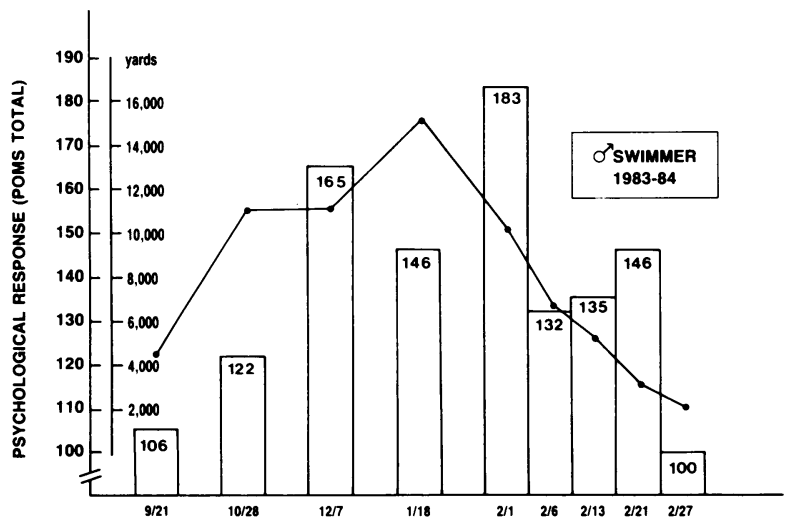

Fig. 17: Global mood state of a selected swimmer during overtraining, staleness, and tapering.

the training micro-cycle which included 15,000 yards per day. It will also be noted that a gradual tapering of the training load from 10,000 yards per day to 2,500 yards per day across four weeks $(5$ micro-cycles) resulted in a reduction in the POMS total to a raw score of 100 just prior to championship competition. This particular case illustrates the rate of recovery in a swimmer who became stale and it suggests that tapering can be effective if there is sufficient time available - four weeks in this case. Otherwise, it would probably be more effective to employ complete rest.

\section{SUMMARY}

It is now widely recognised that aerobic exercise of a vigorous nature is capable of reducing depression in individuals who are suffering from mild or moderate depression, and there is limited evidence that exercise can be an effective adjunct to pharmacologic therapy in severe depressive episodes (Morgan, 1985a; Morgan and Goldston, 1987). It is also known that aerobic exercise leads to various physiological changes such as an increase in maximal aerobic power and reduction in resting and exercise heart rate. It is also widely recognised that training brings about increases in performance. There is a paradox, however, since overtraining can result in the development of mood disturbances and this has been demonstrated in the present paper to occur in competitive swimmers who possessed positive mood states prior to overtraining. Also, non-athletes in the same environment have not been observed to manifest these mood disturbances.

It has also been shown that positive physiological effects can be reversed as well with overtraining. Foster and coworkers for example, reported that speed skaters experienced a significant decrement in maximal aerobic power, as well as performance following overtraining (Foster et al, 1982). Their report is particularly compelling since they employed a measure of "peak" $\mathrm{VO}_{2}$ max rather than a symptom-limited maximum; that is, their measure of maximum can be viewed as a physiological rather than a subjective variable. It is also widely reported that exercise leads to a decrease in resting heart rate but there is evidence that resting heart rate increases with overtraining (Dressendorfer et al, 1985).

The changes in mood state are also associated with various cardiovascular, enzymatic, endocrine, hormonal and hypothalamic changes. When these alterations are viewed in concert with reduction in functional capacity (e.g. reduced $\mathrm{VO}_{2} \max$ ) it is not surprising that performance levels decrease, nor is it surprising that athletes are unable to train at customary levels of intensity. Whilst rest or reduced training seems to be the universal treatment for staleness once it occurs, we propose that performance is most likely to be maximised when training is sufficiently intense to produce desired performance levels, but not so intense as to produce staleness. In other words, we support the use of overtraining in selected micro-cycles but feel the training load should be modified before the state of staleness develops.

It has previously been proposed that performance is inversely correlated with psychopathology (Morgan, 1985b). This mental health model assumes that positive mental health is associated with high performance levels, whereas mood disturbances are predicted to result in performance decrements. Predictions based upon this model have been found to have an accuracy of approximately $80 \%$ in a series of studies (Morgan, 1985b) and research reported on in this paper offers additional empirical support for the model. It is not our intention to question the value of stress markers such as heart rate, blood pressure, lactate, creatine kinase, cortisol or catecholamine levels in monitoring the undesired effects of overtraining. Rather, we have attempted to demonstrate in this paper that monitoring of mood states during a given macro-cycle offers a potential method of quantifying distress and titrating training loads on an individual basis. It is our belief that psychobiologic parameterisation will offer the most effective monitoring strategy and the present report demonstrates the efficacy of including psychologic parameters in modelling efforts designed to prevent the onset of staleness. 


\section{ACKNOWLEDGEMENTS}

The authors wish to acknowledge the expert technical assistance of Michele A. Clermont, MS during the initial phase of this research effort and to thank coaches Jack Pettinger and Carl Johansson and the members of their swimming teams at the University of Wisconsin-Madison who have served as volunteer test subjects over the past ten years.

\section{References}

Akiskal, H. S. and McKinney, W. T., 1975 "Overview of recent research in depression: Integration of ten conceptual models into a comprehensive clinical frame". Archives of General Psychiatry 32: 285-305.

Barron, J. L., Noakes, T. D. and Levy, W., 1985 "Hypothalamic dysfunction in overtrained athletes". Journal of Clinical Endocrinology 60: 803-806.

Bompa, T. O. 1983. Theory and Methodology of Training: The Key to Athletic Performance. Kendall-Hunt Publishing, Dubuque, lowa.

Czajkowski, W., 1982 "A simple method to control fatigue in endurance training". In Exercise and Sport Biology, P. V. Komi (Ed.), Human Kinetics Publishers, Champaign, IL.

Dressendorfer, R. H. and Wade, C. E., 1983 "The muscular overuse syndrome in long-distance runners". Physician and Sportsmedicine 11: 116-126.

Dressendorfer, R. H., Wade, C. E. and Scaff, J. H., 1985 "Increased morning heart rate in runners: A valid sign of overtraining?" Physician and Sportsmedicine 13: 77-86.

Foster, C., Pollock, M., Farrell, P., Maksud, M., Anholm, J. and Hare, J., 1982 "Training responses of speed skaters during a competitive season". Research Quarterly for Exercise and Sport 55: 243-246.

Griffith, C. R., 1926. Psychology of Coaching, Charles Scribner Sons, New York.

Hakkinen, K., Pakarinen, A., Alen, M. and Komi, P. V., 1985 "Serum hormones during prolonged training of neuromuscular performance". European Journal of Applied Physiology 53: 287-293.
Hanson, P. G. Vander Ark, C. R., Besozzi, M. C. and Rowe, G. C., 1982 "Myocardial infarction in a national class swimmer". Journal of the American Medical Association 248: 2313-2314.

Harre, D., 1982 (Chief Editor). Principles of Sports Training, Sportverlag Berlin.

Hickson, R. C., Hagberg, J. M., Conlee, R. K., Jones, D. A., Ehsani, A. A. and Winder, W. W., 1979 "Effects of training on hormonal responses to exercise in competitive swimmers". European Journal of Applied Physiology 41: 211-219.

Karpovich, P. V., 1959. Physiology of Muscular Activity, W. B. Saunders, Philadelphia.

Kereszty, A., 1971 "Overtraining". In Encyclopedia of Sport Sciences and Medicine, Ed. L. A. Larson. Macmillan, New York, pp. 218-222.

Matveyev, L., 1981. Fundamentals of Sports Training. Progress Publishers, Moscow.

Mellerowicz, H. and Barron, D. K., 1971 "Overtraining". In Encyclopedia of Sport Sciences and Medicine, Ed. L. A. Larson. Macmillan, New York pp. 1310-1312.

Morehouse, L. E. and Rasch, P. J., 1958. Scientific Basis of Athletic Training, W. B. Saunders Co., Philadelphia.

McNair, D. M., Lorr, M. and Droppleman, L. F., 1971. Profile of Mood States Manual. Educational and Industrial Testing Service, San Diego.

Morgan, W. P., 1985a "Affective beneficence of vigorous physical activity". Medicine and Science in Sports and Exercise 17: 94-100.

Morgan, W. P., 1985b "Selected psychological factors limiting performance: A mental health model". In Limits of Human Performance. D. H. Clarke and H. M. Eckert (Eds.), Human Kinetics Publishers, Champaign, IL.

Morgan, W. P. and Goldston, S. E. (Eds.), 1987. Exercise and Mental Health. Hemisphere Publishing, Inc., New York.

Morgan, W. P., O'Connor, P. J., Sparling, P. B. and Pate, R. R., 1987 "Psychologic characterization of the elite female distance runner". International Journal of Sports Medicine (In press).

Parmenter, D. C., 1923 "Some medical aspects of the training of college athletes". The Boston Medical and Surgical Journal, 189: 45-50.

Stray-Gundersen, J., Videman, T. and Snell, P. G., 1986 "Changes in selected objective parameters during overtraining". Medicine and Science in Sports and Exercise 18: Supplement 2, 54.

Wolf, J. G., 1971 "Staleness". In Encyclopedia of Sport Sciences and Medicine, Ed. L. A. Larson. Macmillan, New York, pp. 1048-1051.

Title:

\title{
BOOK REVIEW
}

Author:

ORTHOPAEDIC PHYSICAL ASSESSMENT

Publisher:

\author{
D. J. Magee
}

W. B. Saunders 1986

Price: $£ 19.95 \quad$ ISBN 0721618553

The author, in his preface, describes the purpose of the book as the provision of a systematic approach towards comprehensive orthopaedic assessment. He includes history taking, examination procedures and investigation techniques as well as functional anatomy. His forte however, is patient examination, comprehensive in detail and in its wide ranging coverage of the teachings of such recognised exponents as Cyriax, Maitland, McKenzie, Mennell amongst many others.

Although the manuscript was originally developed as part of a manual for physical therapy students at the University of Alberta, the final text is offered to paramedical and medical students as well as to other health professionals. It has been a considered opinion of mine for some time that medical practitioners who pursue their interest in sports medicine often lack the basic essentials of good examination techniques. The physiotherapist on the other hand is often more conscientious in establishing a sound basis for assessment prior to utilising physical therapeutic modalities. I consider, therefore, the book would not be misplaced in the practising Sports Physician's library.

In his regional assessment the author includes the active movement, passive movement and isometric contraction philosophy of Cyriax, the joint play concept of Mennell and co-workers and the quadrant theories of Maitland. By bringing them all together he enables the reader to utilise the text as a suitable reference work in assessment of any part of the musculoskeletal system. There is no grouping of findings into patterns found in clinical syndromes; although the aims of the book do not primarily include interpretation of findings, postulates are made in the less contentious areas. The numerous clinical pictures and diagrams are well presented, although the quality of the X-rays is poor and ancillary investigatory techniques are not covered in any depth.

I commend this book to those medics and paramedics who examine patients and who wish to expand their examining techniques. I enjoyed reading it as the text was clear and set out in an orderly fashion. Readers who come across those concepts such as 'joint play', of which they have limited previous experience are advised to refer to texts by other authors in the list of references. 